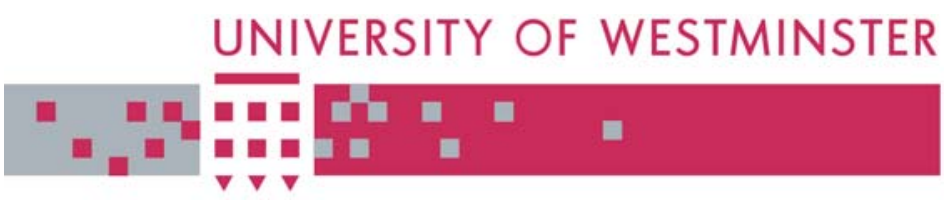

\title{
WestminsterResearch
}

http://www.wmin.ac.uk/westminsterresearch

\section{Distributed VR for Collaborative Design and Manufacturing.}

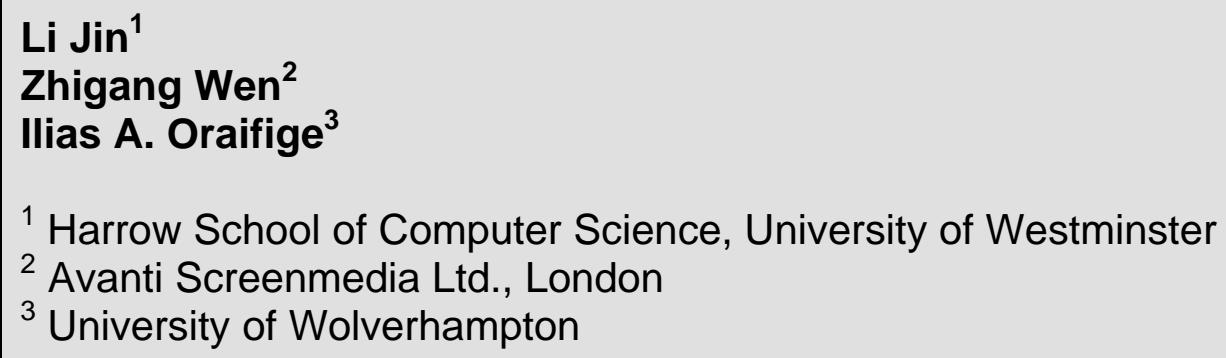

Copyright (c) [2007] IEEE. Reprinted from Proceedings of the 11th International Conference on Information Visualisation: IV '07. IEEE, Los Alamitos, USA, pp. 792-797. ISBN 9780769529004.

This material is posted here with permission of the IEEE. Such permission of the IEEE does not in any way imply IEEE endorsement of any of the University of Westminster's products or services. Internal or personal use of this material is permitted. However, permission to reprint/republish this material for advertising or promotional purposes or for creating new collective works for resale or redistribution must be obtained from the IEEE by writing to pubs-permissions@ieee.org. By choosing to view this document, you agree to all provisions of the copyright laws protecting it.

The WestminsterResearch online digital archive at the University of Westminster aims to make the research output of the University available to a wider audience. Copyright and Moral Rights remain with the authors and/or copyright owners.

Users are permitted to download and/or print one copy for non-commercial private study or research. Further distribution and any use of material from within this archive for profit-making enterprises or for commercial gain is strictly forbidden.

Whilst further distribution of specific materials from within this archive is forbidden, you may freely distribute the URL of the University of Westminster Eprints (http://www.wmin.ac.uk/westminsterresearch).

In case of abuse or copyright appearing without permission e-mail wattsn@wmin.ac.uk. 


\section{Distributed VR for Collaborative Design and Manufacturing}

\author{
Li Jin \\ School of Computer Science \\ Harrow Campus \\ University of Westminster \\ HA1 3TP UK \\ Email: L.Jin02@westminster.ac.uk
}

\author{
Zhigang Wen \\ Avanti Screenmedia Ltd. \\ 74 Rivington Street \\ London EC2A 3AY UK
}

\author{
Ilias A. Oraifige \\ University of Wolverhampton \\ Wolverhampton, WV1 1SB, UK
}

\begin{abstract}
Virtual Manufacturing (VM) applies Virtual Reality (VR) technology to provide a digital manufacturing solution in both $3 D$ visual and interactive ways. It makes $V R$ no longer a state-of-the art but rather an innovation technology to support modern industry. With the rapid growth of network technology and Web $3 D$ graphics techniques, we propose a cost-effective distributed VM system for Small and Medium-sized Enterprises (SMEs) with limited equipment, funds and technical capabilities. The system enables SMEs to perform collaborative tasks including product design, manufacturing and resources sharing through the World Wide Web $(W W W)$ in lower cost. This paper describes the design and critical integration issues of the system and the use of the Web $3 D$ technology $-X 3 D$. It also evaluates the distributed $V M$ system by comparing it to the conventional standalone $C A D / C A M$ system.
\end{abstract}

Keywords: Virtual Manufacturing, World Wide Web, $\mathrm{X} 3 \mathrm{D}$, SMEs.

\section{Introduction}

Virtual Manufacturing (VM) takes advantage of Virtual Reality (VR) technology to provide a digital manufacturing solution in both $3 \mathrm{D}$ visual and interactive ways. It has shown us the powerful potentials of VR by being combined in the latest generations of CAD/CAM tools to aid product development. VM has made VR no longer a state-of-the art but rather an innovation technology to support modern industry [1]. Engineers have applied VM in a wide range of manufacturing applications: product design and prototyping, factory layout design and visualization, assembly process planning and simulations, machinist training, and so forth $[2,3,4,5,6]$. A VM system is based on a virtual environment where a virtual manufacturing world can be modelled, simulated, visualized and even interacted with using immersive devices such as Head Mounted Displays (HMDs), datagloves, force/touch feedback equipment and so on [7]. Because of the complexity of product development in reality, a database involved in a virtual manufacturing environment must be very large in order to create and represent complex scenarios as realistically as possible. In addition, the platform dependence of various VM systems results in incompatibility problems and various 3D graphics data formats also create a barrier for collaboration digital manufacturing.

At present, manufacturers demand to get to market faster with new products. SMEs experience stronger competitive pressures because of the limited equipment, fund and technical capabilities. However, current commercial VM systems are often expensive because of the involvement of high performance graphics accelerators, multiprocessor graphics workstations and some dextrous immersive devices [8]. Thus, most SMEs can't afford such high-cost VR systems and have a clear preference for desktop VR systems over more immersive HMD-based systems. With the rapid growth of network technology, the World Wide Web (WWW) has become a primary medium for information distribution, remote communication and multimedia services via a clientsever approach. In conjunction with Web-based 3D graphics techniques such as the latest X3D and MPEG-4 [9], researchers have applied VR technology to create a virtual heritage, city or shop on the Web $[10,11]$. Therefore, we believe that if a VM system can be accessed on the Web, it's more effective and efficient for SMEs to develop higher-quality products in less time and at lower cost.

In this project, we aim to propose a cost-effective distributed VM system for SMEs. The research was based on the Innovative Product Development Centre (IPDC) at Telford. The centre was established by the University of Wolverhampton in order to assist SMEs to become more competitive by sharing design and manufacturing resources through the WWW. Figure 1 shows the overview of the IPDC. The centre includes a digital manufacturing lab and a manufacturing workshop, which contains several of the latest manufacturing equipments including a rapid prototyping system, reverse engineering instruments, high-speed Computer- 
Numerical-Control (CNC) machines and so on. The digital manufacturing lab consists of VM systems, several common commercial CAD/CAM packages such as PTC's Pro/E Autodesk's AutoCAD, Delcams' Duct $\mathrm{CAD} / \mathrm{CAM}$ system and so on, and a knowledge-based expert system for product assessments. Generally speaking, it's impractical and unnecessary for every SME to purchase all of the above valuable manufacturing resources. Thus, the centre allows registered SMEs to remotely access the product development environment via Internet/Intranet for sharing resources. A client could send a product design along with its specification to the IPDC. After the design has been modified and optimised by experts in this center, the actual prototype can be produced for testing and evaluation. Then the final accepted version is converted into the ComputerNumerically-Controlled (CNC) code for performing the manufacturing process or is sent back to the client. During the procedure, designers can discuss their ideas and requirements with experts by email or networking conference for collaboration working.

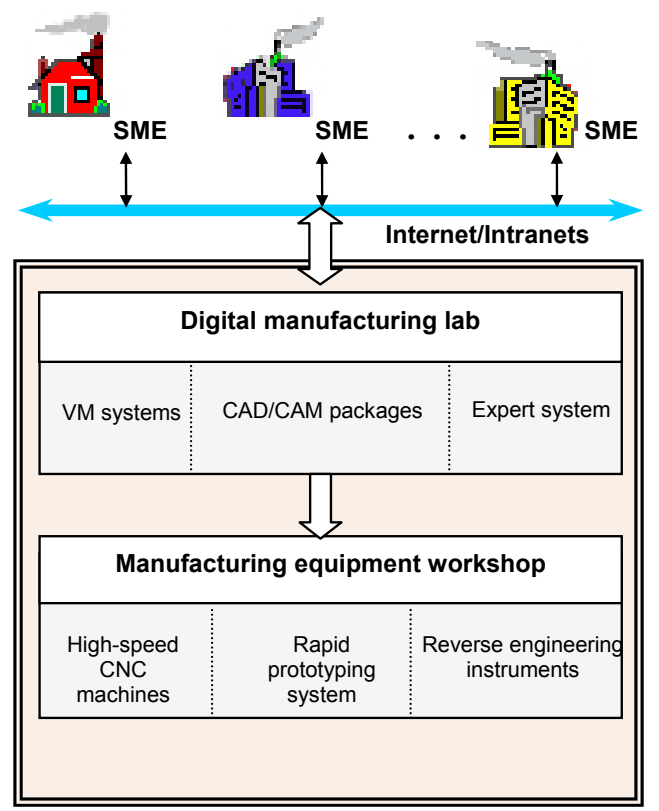

Figure 1 The overview of the Innovative Product Development Centre (IPDC)

This paper describes the design and critical integration issues of the proposed system and the use of the latest Web-based 3D technology - X3D. The following section presents a distributed Web-based VM system using X3D to create a sharing environment for design and manufacturing applications. Section 3 describes the critical integration issues in such a distributed VM system, which includes 3D geometric modelling, VR simulation engine, and real time interaction modes. Section 4 discusses the overall evaluation of the proposed approach by comparing to the conventional stand-alone CAD/CAM systems. Finally, section 5 presents the future work and draws some brief conclusions.

\section{Distributed Web-based VM system Using X3D}

VM systems, as main components in the IPDC, consist of a fundamental system and a Web-based system for the Internet access. The fundamental system is an immersive VR system. It supports not only the common monitors but also stereoscopic large-screen projection.

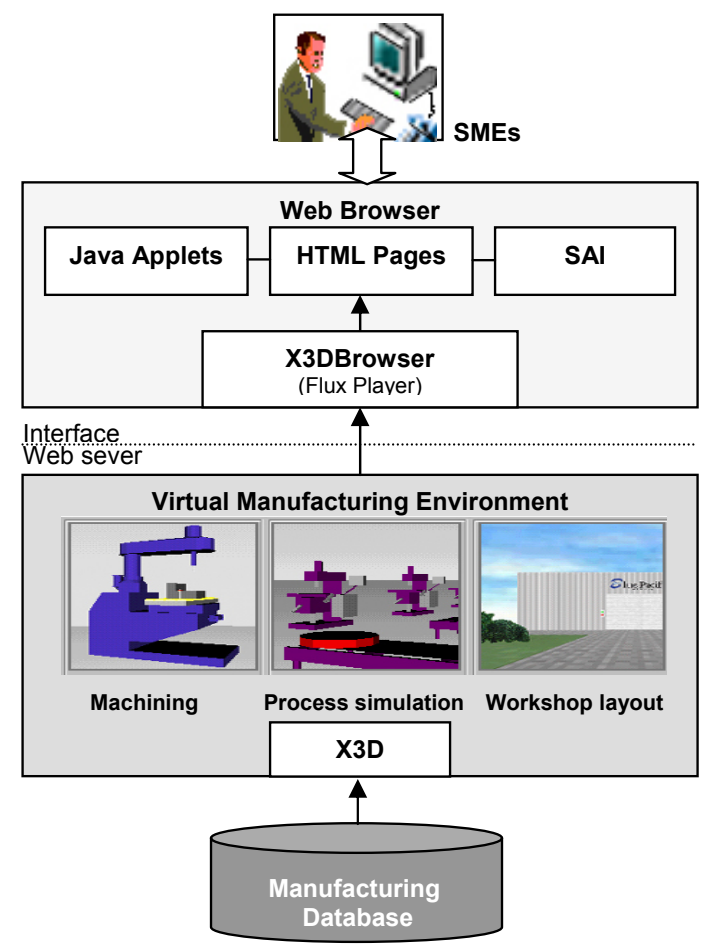

Figure 2 The framework of Web-based VM system

The engineers use a mouse, keyboard and dataglove to immerse in a virtual manufacturing environment. Since most SMEs do not need the full bandwidth of immersion, a Web-based VM system was designed as a costeffective VR approach for SMEs to use for aiding product development.

This section presents a Web-based VM system which uses the WWW as the backbone to create a sharing environment for its distribution. This system allows multiple users (engineers, machinists and trainees etc.) to access and explore some virtual manufacturing applications at any sites or any time without specialised computer platforms or software. Figure 2 shows the system's framework. 
The system consists of a digital manufacturing database, a virtual manufacturing application environment and an interface for the Internet access. The database includes a Virtual Machine library (VM Lib) along with design and manufacturing data. All 3D modelling data are converted into X3D format. The X3D as the Web 3D International Standard (ISO/IEC 19775) using Extensible Markup Language (XML) provides a standardized, portable, and platform-independent way to render dynamic interactive $3 \mathrm{D}$ scenes across the Internet [12]. It represents static and animated dynamic 3D and multimedia objects with hyperlinks to other media such as text, images, sounds, and videos. The characteristic of X3D is consistent with that of Hypertext Markup Language (HTML). Therefore, an X3D world through an $\mathrm{X} 3 \mathrm{D}$ browser such as Flux Player can be seamlessly embedded into the normal Web browser such as Microsoft Internet Explorer (IE). The user accesses and navigates an $\mathrm{X} 3 \mathrm{D}$ world downloaded from the Internet server. In this way, X3D allows the delivery of such an interactive VM system through the Internet to reach potentially large number of users worldwide at any time.

By reducing costs and cycle time, the distributed VM system is able to enhance and even accelerate the major activities of manufacturing engineering, including: concept development and simulation, optimising assembly lines and workshops design, integrating labour and equipment, etc. Three major manufacturing applications including machining, process planning and workshop layout are prototyped based on the proposed approach in the Web-based virtual manufacturing environment.

- Machining-visualizes and analyzes the functionality of a machine. The application emulates components, tools, controllers and workpiece along with machining process. It aids SMEs to preview simulation and analysis. It aids SMEs in reducing risk and saving time before establishing a real process flow.

- Workshop layout - in order to get a cost-effective and safe manual Workplace, the application allows SMEs to design work places and equipment layout before the start of production. An optimal workshop layout design according to the rules of ergonomics could maximise the efficiency of both workers and equipment.

In addition, the virtual manufacturing environment relies on a Web-based graphical user interface (GUI) for Internet accesses. Java, JavaScript and X3D SAI (Scene Access Interface) can be used to create a connection between an external environment and the X3D scene [13]. The Web-based GUI integrated X3D browser, Java, JavaScript and SAI allows multiple users to interact and communicate with virtual manufacturing applications over the Internet.

\section{Critical integration issues}

The Web-based VM system allows multiple users to access integrated manufacturing environments. Such integrated environments simulate actual manufacturing applications which could be interacted with through the WWW. The critical integration issues in such a distributed Web-based VM system include 3D geometric modelling, VR simulation engine, and real time interaction mode.

\subsection{Modelling 3D virtual machines}

In a VM system, a 3D geometrical machine model created according to a real one is used to create a virtual manufacturing world. A real machine consists of many components, which are made up of different parts.
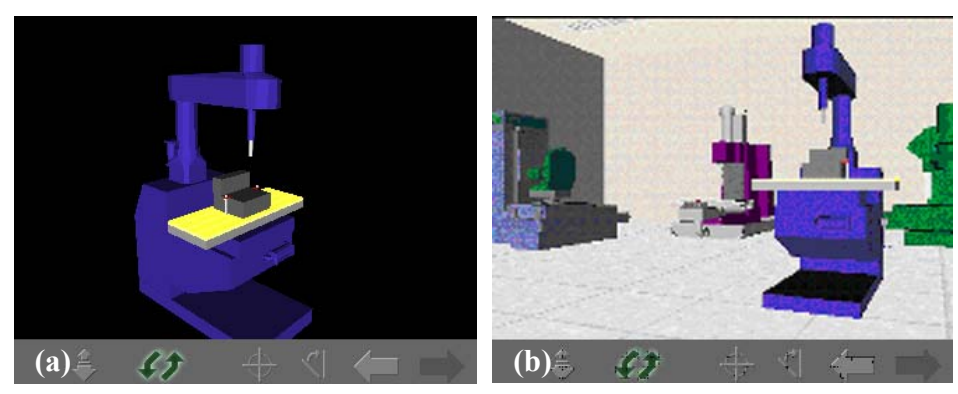

Figure 3. Reusing a machine model in various manufacturing applications. (a) A mill model in the machining application. (b) The reuse of a mill model in the workshop layout application.

and explore a new or valuable machine before its purchase. Engineers and machinists can be trained on the processes of a machine set-up, tools change and production programs.

- Process simulation - simulates a process flow in a virtual workshop. It offers an opportunity for SMEs to get the solution of choice for process flow
For example, a conventional milling machine comprises a base, a working table, a head, main shaft, a servo motor and a spindle motor, etc. In this project, a machine model is constructed according to a part-component-machine procedure and then imported them into the Virtual Machine Library (VM Lib). X3D geometric modelling is mainly based on geometric primitives such as cubes, 
spheres, cylinders, cones, and so on. It is basically used to model some components whose shapes are regular. The various modelling methods are integrated to shorten the system development cycle. For example, some common machine models such as mills and lathes are exported and converted into X3D format from a machine library provided by some professional CAD/CAM packages such as Deneb's Virtual NC and QUEST. In addition, a professional geometric modelling package such as Kinetix' 3D Studio Max or Pro/Engineer is used to create some new types of machines. Similarly, the model files are converted into X3D format via a converter. However, the model's size could be very large because each polygon is converted into an IndexedFaceSet node description. In order to improve download speed and navigation performance, a model needs to be optimised by reducing the number of polygons required.

X3D files exist in a scene graph hierarchical structure. Models are subparts of the world. Such a structure makes it easy to create large worlds or complicated scenarios, which reuse subparts or inherit attributes and behaviours from subparts. As a result, not only a machine model but also its components or parts can be reused in various manufacturing applications. For example, Figure 3 a shows a milling machine model used in the machining application. Meanwhile, the model can be reused as a subpart in a workshop layout application shown in Figure 3b.

\subsection{VR simulation engine}

A dynamics machine and a process flow need to be simulated according to the level of detail required. The VR simulation engine plays an important role in generating dynamic simulations. In this project, the following mechanisms of X3D [14] have been used to develop interactive dynamic behaviour simulations in the distributed Web-based VM system. different types of interpolators: PositionInterpolator, OrientationInterpolator, CoordinateInterpolator, and so forth. Each one animates a different field type. For example, the PositionInterpolator node is used to animate a cutting tool's position along a motion path. Thus, a PositionInterpolator node and an OrientationInterpolator node are combined to be used to produce typical animated object motion. The first moves the object along a motion path, while the latter rotates the objects as it moves.

- Sensor nodes. Detect and sense changes to the state of an input device, changes in time, or changes related to the motion of the viewer or objects in a virtual world. They include TouchSensor, CylinderSensor, SphereSensor, TimeSensor, VisibilitySensor, and so forth. The TimeSensor node generates events as time passes and is the basis for all animated behaviors.

- Script nodes. Define arbitrary behaviours by binding the Java and JavaScript languages. Although interpolator nodes don't support spline curve interpolation, a script can directly implement the mathematics of the curve interpolation.

Generally, Interpolator nodes are essentially builtin scripts that perform simple animation calculations. They are usually combined with a TimeSensor node in the scene graph to make objects move. For example, PositionInterpolator node and TimeSensor node are employed to define the moving of the working bed of a mill along $x$-axis in specified time (see Figure $4 \mathrm{a}$ and 4b).

In addition, Physical Based Modelling (PBM) is an important method to simulate animation and dynamic behaviours in VR systems [15]. It can be integrated in the VR simulation engine to generate highly realistic animations based on physical dynamics implemented in Java. Since some professional manufacturing simulation packages such as Delmia's QUEST can directly emulate
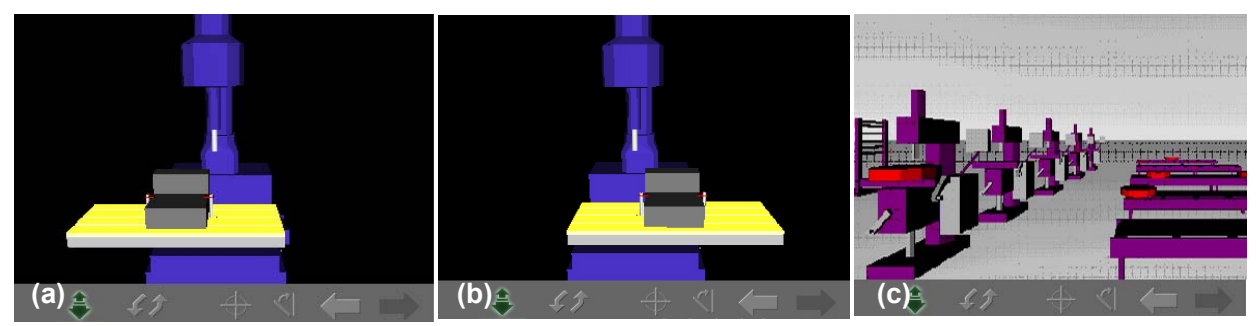

Figure 4 The simulation of a dynamic machine and a process flow in X3D

(a) (b) The simulation of a mill's working bed moving along $x$-axis.

(C) A view of the simulation of a process flow through Flux Player

- Interpolator nodes. Perform keyframe animations. According to a list of specified keyframe values and times, the X3D browser will automatically interpolate the "in-betweens". There are several real-world system behaviors, we intend to couple its simulation capabilities to X3D worlds via a translator. Figure $4 \mathrm{c}$ shows such a simulation of a process flow through X3D browser. 


\subsection{Real time interaction mode}

The distributed VM system provides users with an intuitive Web-based GUI for Internet access and real time interaction. A hierarchy-structural interface through multiple HTML pages is prototyped in this project as shown in Figure 5. The X3D worlds are individually embedded in multiple HTML pages. Each world represents a selected manufacturing application. The Web-based GUI integrates X3D browser, Java applet and X3D SAI to allow users to interact with applications in real time. The Scene Access Interface (SAI) provides the mechanism to communicate between Java applet and an X3D scene graph. Each application's HTML page contains Java applets running along side the X3D worlds. As an input tool, the Java applet can manipulate and update an X3D scene via the SAI and communicate between the client and server.

The method takes advantage of the event-routing mechanism of X3D. Some X3D nodes generate events in response to environmental changes or user interaction. These events can be propagated to effect changes in other nodes. Once generated, events are sent to their routed destinations in time order and processed by the receiving node in $\mathrm{X} 3 \mathrm{D}$. This processing can change the state of the node, generate additional events, or change the structure of the scene graph in an X3D world. The ideal event model processes all events instantaneously in the order that they are generated. Using the Web-based GUI, the Java applets routes input values from users or updated data from the server to their destinations via SAI. Moreover, it allows events to be processed based on the order of user actions or the elapsed time between events while keeping several related X3D scenes synchronised.

\section{Evaluation}

The Web-based VR approach provides engineers and designers with the capability to visualise, explore, manipulate and interact with applications in a distributed virtual environment to support design and manufacturing for collaborative product development via the Internet. This section evaluates the proposed approach in this research project. Compared to a conventional stand-alone CAD/CAM system, the proposed Web-based VM system has considerable advantages:

- $3 D$ visual: Quickly produces various X3D scenes in visual 3D way by manipulating 3D objects.

- Distributed. Permits the sharing of a large virtual manufacturing database on a Web server among distributed SMEs through the WWW.

- Real-time interaction: Allows distributed Webbased users to interact with the virtual environments in real-time.

- Platform-independence. Performs on a wide variety of computing platforms. Therefore, SMEs take advantage of their already existing computer facilities.

- Standardization: is compatible with Web-based 3D international standard (ISO/IEC 19775) -X3D.

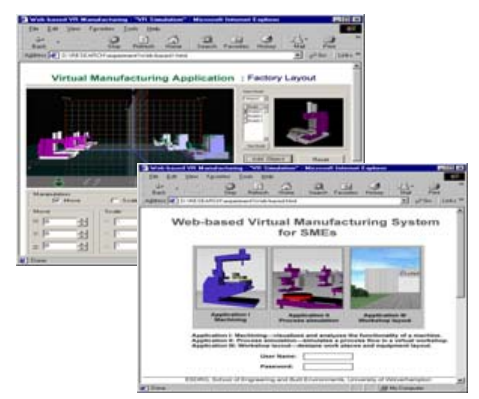

Fiqure 5 The Web-based GUI

- Low-cost. No licensed software and additional system maintenance are required for SMEs.

- Scalability. Handles large numbers of SMEs in a large-scale virtual environment.

- Extensibility. Enables arbitrarily large dynamic virtual manufacturing worlds and hyperlinks to other engineering information system according to SMEs' requirements.

- Collaboration. Supports multiple users' access. A cluster of co-operated SMEs could communicate with each other for collaboration working.

\section{Conclusions and future work}

The cost-effective Web-based VM system allows engineers and designers to visualize, explore, manipulate and interact with manufacturing applications in a distributed virtual environment. It allows SMEs to easily reuse and widely share the design and manufacturing resources through the WWW. By reducing costs and cycle time of product development, such a distributed VM system will accelerate the major activities of manufacturing including: manufacturing process concept development and simulation, optimising assembly lines and workshops design, integrating labour and equipment, and so forth.

Furthermore, we note that in reality, people always play an essential role in every practical design and manufacturing activity. Therefore, people such as workers beside an assembly line need to be represented in a virtual environment in some applications. In particular, with the introduction of people models to a workshop layout application, a layout design can be enhanced according to ergonomics rules. Such enhancement will aid the improvement of work efficiency and the achievement of the full performance of equipment. However, the integration of virtual humans may result in overloading polygon rendering on the limited network bandwidth because of its large data. Since the proposed VR-based simulation system is 
intended to be distributed across the Internet, navigation speed must be considered to keep the sense of presence. Thus, more effective methods [16, 17] need to be explored in order to optimise the labour integration in the Web-based VM system in future work. Furthermore, multimedia issues such as video and network meeting modules could be incorporated to improve real-time discussion for collaboration working in order to enhance the Web-based system on a broad base of manufacturing and industrial applications for e-service via the Internet.

\section{References}

[1] Weyrich.M, Drews.P. An interactive environment for virtual manufacturing: the virtual workbench. Journal of Computers in Industry. Vol. 38 No.1. 1999. Elsevier Science Publishers. PP5-15.

[2] Zorriassatine.F, Wykes.C, Parkin.R, Gindy.N. A survey of virtual prototyping techniques for mechanical product development. Journal of Engineering Manufacture. Vol. 217 No.4. 2003. PP513-530.

[3] Bao.J, Jin.Y, Gu.M, Yan.J, Ma.D. Immersive virtual product development. Journal of Materials Processing Technology, 2002. PP592-596.

[4] Mancini.F, Vigano.G, Liao.Z, Sacco.M, Boer.C. The virtual factory a semi-immersive interactive 3D environment. Proceedings of the 2004 Summer Computer Simulation Conference, 2004. San Jose, USA. PP502-506.

[5] Choi.B, Park.M, Ryu.H. Virtual factory simulator for line prototyping. Journal of Advanced Manufacturing Systems Vol. 3 No. 1. 2004. PP5-20.

[6] Mok.S, Wu.C, Lee.D. Modelling automatic assembly and disassembly operations for virtual manufacturing. IEEE Transaction on Systems, Man and Cybernetics, Part A, Vol. 31 No. 3. 2001. PP223 - 232.

[7] Murray.N, Fernando.T. An immersive assembly and maintenance simulation environment. Proceedings of IEEE International Symposium on Distributed Simulation and Real-Time Applications, 2004. PP159166.

[8] Kim.Y, Yang.J, Han.S. A multichannel visualization module for virtual manufacturing, Journal of Computers in Industry. Vol. 57 No. 7. 2006. Elsevier Science Publishers. PP653-662.

[9] Rhyne.T.M. Internetworked 3D Computer Graphics: Overcoming Bottlenecks and Supporting Collaboration.
ACM SIGGRAPH 2000 Course \#28 Notes. http://www.crcg.edu/events/siggraph/ sig00/ theresa/ overview-netgraf3.html.

[10] Farrimond.B, Hetherington.R. Compiling 3D models of European heritage from user domain XML. Proceedings of the $9^{\text {th }}$ International Conference on Information Visualisation, 2005. PP163-171.

[11] Marvie.J, Bouatouch.K, A VRML97-X3D extension for massive scenery management in virtual worlds. Proceedings of the $9^{\text {th }}$ International conference on 3D Web Technology, 2004. ACM Press. PP145-153.

[12] Chittaro.L, Ranon.R. Using the X3D language for adaptive manipulation of $3 \mathrm{D}$ Web content. Proceedings of AH-2004: 3rd International Conference on Adaptive Hypermedia and Adaptive Web-based Systems, 2004. Springer-Verlag, Berlin. PP287-290.

[13] Extensible 3D (X3D) SAI Specifications. Web 3D Consortium. http://www.web3d.org/x3d/specifications/ ISO-IEC-19775-X3DAbstractSpeci fication/

[14] Extensible 3D (X3D) ISO/IEC 19775:2004 Specifications. Web 3D Consortium. http://www.web3d.org/x3d/specifications/ISO-IEC19775-X3DAbstractSpecification/

[15] Allard.J, Raffin.B. Distributed physical based simulations for large VR applications. Proceedings of IEEE Virtual Reality Conference, 2006. PP89-96.

[16] Ieronutti.L, Employing virtual humans for education and training in X3D/VRML worlds. Proceedings of LET-Web3D 2004: 1st International Workshop of Web3D Technologies in Learning, Education and Training, 2004. PP23-27.

[17] Buttussi.F, Chittaro.L, Nadalutti.D. H-Animator: A visual tool for modelling, reuse and sharing of X3D humanoid animations. Proceedings of Web3D 2006: 11th International Conference on 3D Web Technology, 2006. ACM Press. PP109-117. 